\title{
Research Article \\ Effect of Interaction between Early Menarche and Genetic Polymorphisms on Triglyceride
}

\author{
Ho-Sun Lee $\mathbb{D}^{1},{ }^{1}$ Sangseob Leem $\mathbb{D}^{1},{ }^{1}$ Bermseok Oh $\mathbb{D}{ }^{2}$ and Taesung Park $\mathbb{D}^{1}$ \\ ${ }^{1}$ Interdisciplinary Program in Bioinformatics and Department of Statistics, Seoul National University, 1 Kwanak-Ro, Kwanak-gu, \\ Seoul 151-747, Republic of Korea \\ ${ }^{2}$ Department of Biochemistry and Molecular Biology, School of Medicine, Kyung Hee University, Seoul 02447, Republic of Korea
}

Correspondence should be addressed to Taesung Park; tspark@stats.snu.ac.kr

Received 21 July 2018; Revised 6 November 2018; Accepted 10 November 2018; Published 25 February 2019

Guest Editor: Susana Novella

Copyright (c) 2019 Ho-Sun Lee et al. This is an open access article distributed under the Creative Commons Attribution License, which permits unrestricted use, distribution, and reproduction in any medium, provided the original work is properly cited.

Early menarche has been associated with increased risk of metabolic syndrome. Therefore, investigating the association of each component of metabolic syndrome with age at menarche, and interactions between them, might lead to a better understanding of metabolic syndrome pathogenesis. In this study, we evaluated age at menarche for risk of metabolic syndrome and associations with its components. As a result, the risk of MetS incidence was significantly increased only at $\leq 12$ years of age at menarche $(\mathrm{OR}=1.91, P<0.05)$. Women with early menarche $(\leq 12$ years) had significantly higher levels of triglycerides $(\beta$ coefficient $=37.83, P=0.02)$. In addition, hypertriglyceridemia was significantly increased at early menarche with $1.99(95 \% \mathrm{CI}$ : 1.16-3.41, $P<0.01)$. With GWAS-based pathway analysis, we found the type 2 diabetes mellitus, stress-activated protein kinase signaling, and Jun amino-terminal kinase cascade pathways (all nominal $P<0.001$, all FDR $<0.05$ ) to be significantly involved with early menarche on triglyceride levels. These findings may help us understand the role of early menarche on triglyceride and interaction between gene and early menarche on triglyceride for the development of metabolic syndrome.

\section{Introduction}

Menarche, defined as the first menstrual period in a woman's life, is a marker of female puberty and the onset of ovarian and other endocrine functions related to reproductive capacity. Age of menarche has declined over the last several decades through improvement of socioeconomic conditions or exposure to environmental chemicals and received a great deal of attention as having important health implications [1]. Age at menarche (AAM) can be a risk factor for disease, and there were several studies on the interaction between AAM and genetic factors especially for breast cancer susceptibility [2-4]. Early menarche is often defined as menarche before the age of 12 years ( $\leq 11$ years old), but some investigators base definition on menarche at $\leq 12$ years [5]. A number of studies have reported associations between early menarche onset and intermediate quantitative traits such as increased blood glucose levels [6], impaired glucose tolerance [7], and insulin resistance [8], further supporting a link between early pubertal development and an adverse metabolic profile.
Others have reported adverse metabolic consequences (e.g., diabetes) arising from early sexual maturation $[9,10]$. One possible mechanism for this association is a direct link between puberty timing and glucose regulation, supported by the insulin-sensitizing agent metformin delay of menarche in girls with precocious puberty [11]. It is conceivable that part of the association between menarche onset and type 2 diabetes risk is explained by increased adiposity $[9,10]$. However, these data are not consistent, and the relationship between AAM and metabolic components remains poorly understood. Metabolic syndrome (MetS) is considered a worldwide epidemic. In general, the International Diabetes Federation estimates that one-quarter of the world's adult population has MetS [12]. Higher socioeconomic status, sedentary lifestyle, and high body mass index (BMI) significantly associate with MetS. Data from the National Health and Nutrition Examination Survey (NHANES, 1999-2006) reported that nearly $34 \%$ of all U.S. adults, and $50 \%$ of those aged 60 years or older, were estimated to have MetS [12]. The prevalence of MetS in Korea has also been steadily increasing 
in recent years, from $24.9 \%$, in 1998 , to $31.3 \%$, in 2007 ; this prevalence is relatively high compared to those of other Asian countries [13]. MetS is characterized by the clustering of five pathological traits, including large waist circumference, hypertriglyceridemia, low-density lipoprotein (LDL) cholesterol level, hypertension, and hyperglycemia. However, mechanisms and differences, correlated with the relative risk of susceptibility to MetS, remain poorly characterized.

To date, genome-wide association studies (GWAS) have identified several susceptibility regions and genes for MetS and its component phenotypes $[14,15]$. Since the first report from a GWA study of obesity, an increasing number of genetic factors have been shown to associate with several traits of obesity and MetS. Despite the success of recent GWAS, the identified variants explain only a small proportion of the heritability of most complex diseases [16]. Heritability estimates of MetS range from approximately 10 to $30 \%$ [17] among individuals; therefore, implicated genes may interact synergistically with environmental factors, in the pathogenesis of MetS.

Here, we investigated the role of AAM on MetS components for MetS risk. Specifically, we examined the association between AAM and levels of individual component of MetS such as triglyceride (TG). Furthermore, we evaluated GWAS-identified genetic variants for interaction effects with MetS components using a pathway-based analysis and characterized the role of AAM in the pathogenesis of MetS.

\section{Methods}

2.1. Study Design and Participants. This study, an ongoing prospective investigation, is a population-based cohort included in the Korean Genome and Epidemiology Study (KoGES) [18]. Originally, 5018 woman participants, aged 40 to 69 years, were recruited from the Korean rural (Ansung) and urban (Ansan) communities at baseline, as part of the Korean Association Resource Project (KARE). From 2007 to 2008, the samples were scrutinized for quality control purposes, and 3493 participants remained. We excluded those with reported AAM of $\leq 10$ years $(n=3)$ or $\geq 19$ years $(n=272)$ or missing $(n=41)$, probably due to recall error or the presence of a comorbid pathological condition. Finally, information was available from 3180 women for the study of AAM.

These cohorts included a standardized health interview, using well-established questions, to determine the demographic and socioeconomic characteristics of all subjects, including menstruation history. AAM was defined as the age of the first menstrual period. Women reported their AAM in single years and were classified for this study as having an $\mathrm{AAM}$ of $\leq 12,13,14,15,16$, or $\geq 17$ years [19]. Comprehensive health examinations, including evaluation of anthropometric indexes, measurement of blood pressure, questionnaire-based interviews, and collection of biospecimens for assays (e.g., C-reactive protein), were conducted biennially by health professionals who were trained with a standardized protocol. Women who smoked regularly during the previous 12 months were classified as current smokers.
Levels of physical activity were semiquantitatively assessed using a questionnaire previously validated against objective methods [20] and were coded as inactive, moderately inactive, moderately active, and active. Educational attainment was categorized into three groups: less than 7 years (elementary school graduates), 7-9 years (middle school graduates), and more than 10 years (high school graduates). Monthly household income was also categorized into three groups: less than $\$ 1000$ USD (in 2014), $\$ 1000-2000$, and $\geq \$ 2000$ [21]. In addition, we performed the additional analysis for oxidative stress-related exposure with OBS score and inflammatory marker with C-reactive protein (CRP) at each AAM $[22,23]$. Written informed consent was obtained from all participants at the KoGES. This study was approved by the Institutional Review Board of Seoul National University.

\subsection{Definition of the Metabolic Syndrome and Its Components.} We used the definition of MetS proposed by the criteria of the National Cholesterol Education Program Adult Treatment Panel III (NCEP-ATP III) guidelines [24]. As detailed in our study, participants having three or more of the following criteria were defined as having MetS, except for the determination of central obesity. Waist circumference cut-off values were used based on guidelines from the Korean Society for the Study of Obesity [25]: (1) central obesity, given as waist high circumference $(\geq 85 \mathrm{~cm})$; (2) high concentration of serum triglycerides $(\geq 150 \mathrm{mg} / \mathrm{dl})$; (3) low concentrations of serum HDL cholesterol (<50 mg/dl); (4) hypertension (systolic/diastolic pressure $\geq 130 / 85 \mathrm{mmHg}$ ) or antihypertensive medication; and (5) high concentrations of fasting glucose $(\geq 100 \mathrm{mg} / \mathrm{dl})$ or antidiabetic medication.

2.3. Genotyping. The data used for GWAS was obtained from KoGES, which was conducted by the Korean National Institute of Health. DNA samples were isolated from the peripheral blood of all participants and genotyped, using the Affymetrix Genome-Wide Human SNP array 5.0 (Affymetrix Inc., Rockville, MD, USA). Quality control procedures were adopted, such as missing genotype frequency $>0.5 \%$ and minor allele frequency $(\mathrm{MAF}) \leq 0.05$. After sample and genotype quality controls, 344,396 SNPs for 3180 individuals were available in the KARE database $[26,27]$. The KARE data used to support the findings of this study are restricted by the Institutional Review Board of the Korean National Institute of Health, who can contact at National Biobank of Korea (http://koreabiobank.re.kr, 82-1661-9070).

2.4. Statistical Analysis. For demographics and characteristics of subjects, data were presented as means and standard deviations for continuous variables, or a percentage (\%), for categorical variables (Table 1). Differences among subjects in different groups were detected using the Kruskal-Wallis test, for continuous variables, and the chi-square test, for categorical variables.

At first, logistic regression analyses were performed to determine the odds ratios (ORs) of MetS (Figure 1), and its five components, depending on each year of menarche, with 16 years of age as a reference group (Figure 2). Logistic regression models were adjusted for age, area, income, education, 
TABLE 1: Characteristics of study subjects.

\begin{tabular}{|c|c|c|c|c|c|c|c|c|}
\hline & \multicolumn{6}{|c|}{ Age at menarche (years) } & \multirow{2}{*}{ All } & \multirow{2}{*}{$P^{\mathrm{b}}$} \\
\hline & $\leq 12$ & 13 & 14 & 15 & 16 & $\geq 17$ & & \\
\hline Women, $n(\%)$ & $77(2.4)$ & $269(8.5)$ & $531(16.6)$ & $669(21.1)$ & $689(21.7)$ & $945(29.8)$ & 3180 & \\
\hline Age at enrolment (years) & $51.25 \pm 6.92$ & $52.58 \pm 7.74$ & $53.33 \pm 8.47$ & $54.92 \pm 8.80$ & $56.94 \pm 8.82$ & $59.18 \pm 8.49$ & $56.07 \pm 8.88$ & $<0.001$ \\
\hline \multicolumn{9}{|l|}{ Area, $n(\%)$} \\
\hline Rural (Ansung) & $26(34)$ & $83(31)$ & $199(37)$ & $339(51)$ & $412(60)$ & $640(68)$ & 1699 & $<0.001$ \\
\hline Urban (Ansan) & $51(66)$ & $186(69)$ & $332(63)$ & $330(49)$ & $277(40)$ & $305(32)$ & 1481 & \\
\hline BMI $\left(\mathrm{kg} / \mathrm{m}^{2}\right)$ & $25.55 \pm 3.37$ & $24.62 \pm 3.42$ & $24.78 \pm 3.07$ & $24.72 \pm 3.20$ & $24.86 \pm 3.16$ & $24.89 \pm 3.12$ & $24.80 \pm 3.16$ & 0.848 \\
\hline Current smokers, $n(\%)$ & $0(0)$ & $3(1.1)$ & $9(1.7)$ & $11(1.6)$ & $9(1.3)$ & $27(2.9)$ & $59(1.8)$ & 0.177 \\
\hline Alcohol consumption $(\mathrm{g} / \mathrm{l})$ & $3.52 \pm 11.18$ & $1.51 \pm 5.36$ & $1.49 \pm 5.28$ & $1.40 \pm 6.85$ & $1.41 \pm 6.16$ & $1.28 \pm 4.96$ & $1.44 \pm 5.96$ & 0.068 \\
\hline Age at menopause & $48.26 \pm 3.83$ & $47.52 \pm 5.61$ & $47.60 \pm 4.62$ & $47.87 \pm 5.40$ & $47.83 \pm 5.11$ & $47.90 \pm 5.24$ & $47.82 \pm 5.16$ & 0.718 \\
\hline OBS score & $9.34 \pm 2.41$ & $9.39 \pm 2.43$ & $9.53 \pm 2.56$ & $9.19 \pm 2.47$ & $8.85 \pm 2.53$ & $8.91 \pm 2.50$ & $9.10 \pm 2.51$ & 0.698 \\
\hline CRP (mg/dl) & $1.04 \pm 1.34$ & $1.22 \pm 1.10$ & $1.33 \pm 1.24$ & $1.35 \pm 1.23$ & $1.40 \pm 1.27$ & $1.53 \pm 1.44$ & $1.41 \pm 1.30$ & 0.07 \\
\hline Education, $n(\%)$ & & & & & & & & $<0.001$ \\
\hline Less than elementary school & $7(9.1)$ & $20(7.4)$ & $55(10.4)$ & $95(14.2)$ & $138(20.1)$ & $225(23.8)$ & $540(17)$ & \\
\hline Middle school graduate & $24(31.2)$ & $111(41.3)$ & $185(35)$ & $305(45.6)$ & $384(55.8)$ & $575(60.8)$ & $1584(50)$ & \\
\hline High school and above & $46(59.7)$ & $138(51.3)$ & $289(54.6)$ & $269(40.2)$ & $166(24.1)$ & $141(24.5)$ & $1049(33)$ & \\
\hline Income, $n(\%)$ & & & & & & & & $<0.001$ \\
\hline$<100^{\mathrm{a}}$ & $16(20.8)$ & $61(22.7)$ & $138(26.4)$ & $243(36.6)$ & $305(44.8)$ & $502(53.1)$ & $1265(40.2)$ & \\
\hline $100-200$ & $23(29.9)$ & $59(21.9)$ & $112(21.5)$ & $144(21.7)$ & $156(22.9)$ & $220(23.3)$ & $714(22.7)$ & \\
\hline$\geq 200$ & $38(49.4)$ & $149(55.4)$ & $272(52.1)$ & $277(41.7)$ & $220(32.3)$ & $214(22.6)$ & $1170(37.1)$ & \\
\hline
\end{tabular}

Values are expressed as means \pm SDs (standard deviations) or number (\%). BMI: body mass index; OBS: oxidative balance score; CRP: C-reactive protein; ${ }^{\mathrm{a}} 10^{4}$ KRW: equivalent with 1000 US dollar in 2014. ${ }^{\mathrm{b}} \mathrm{P}$ value was examined by the Kruskal-Wallis test or chi-square test.

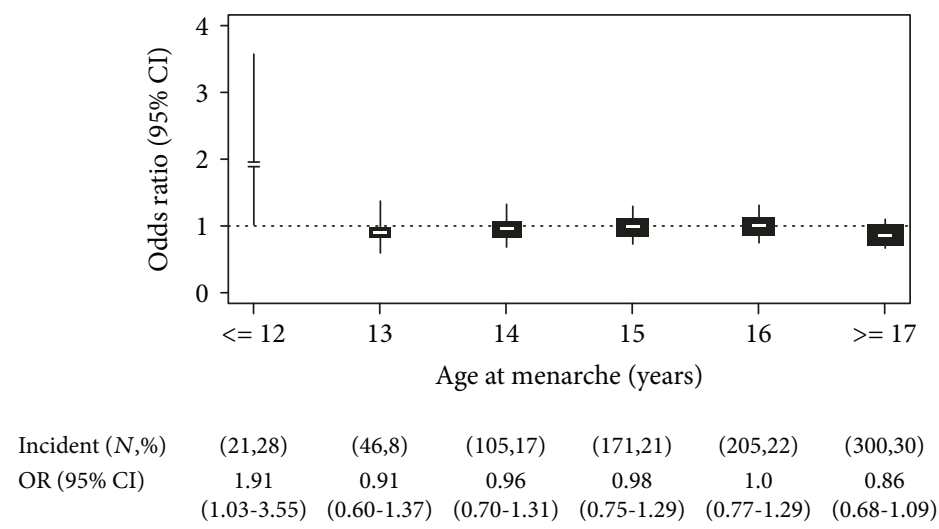

Figure 1: Risk of MetS in each age of menarche. Odds ratio (OR) and 95\% confidence interval (CI) of Mets by each age at menarche. $N$ is the number of incidence in each age of menarche; \% is the percent of incidence in each age of menarche. The size of rectangle represents the number of samples. The vertical line indicates the overall OR on each age of menarche. ORs were adjusted for area, age, income, education, and C-reactive protein levels. The reference category was menarche at 16 years of age.

and CRP levels. Next, we evaluated beta coefficients for each year of menarche, using linear regression analyses for individual components of MetS, with 16 years of age as a reference group (Table 2). This reference group was chosen because it covers the median of AAM (median age $=16.0)$ [28].

To test the interactions between SNPs and AAM, we performed interaction tests for identifying genetic variants associated with MetS and its characteristic components. In addition, we performed the joint test of the main and interaction effects with one degree of freedom (df). We then used the following equation:

$$
Y_{i}\left(\text { or } \log \text { it } p_{i}\right)=\beta_{0}+\beta_{1} E_{i}+\beta_{\text {main }} G_{\mathrm{i}}+\beta_{\text {int }} G E_{\mathrm{i}} \text {, }
$$

where $Y_{i}$ is the MetS component for individual $i$ and $p_{i}=P_{r}$ $\left(Y_{i}=1\right)$ of MetS for a binary trait. We then used the Wald test statistic to report the results of the joint $\left(P_{\text {joint }}\right)$ test, with two $\mathrm{dfs}$ and the gene-risk factor $\left(P_{\text {int }}\right)$ with one df, referring to their 


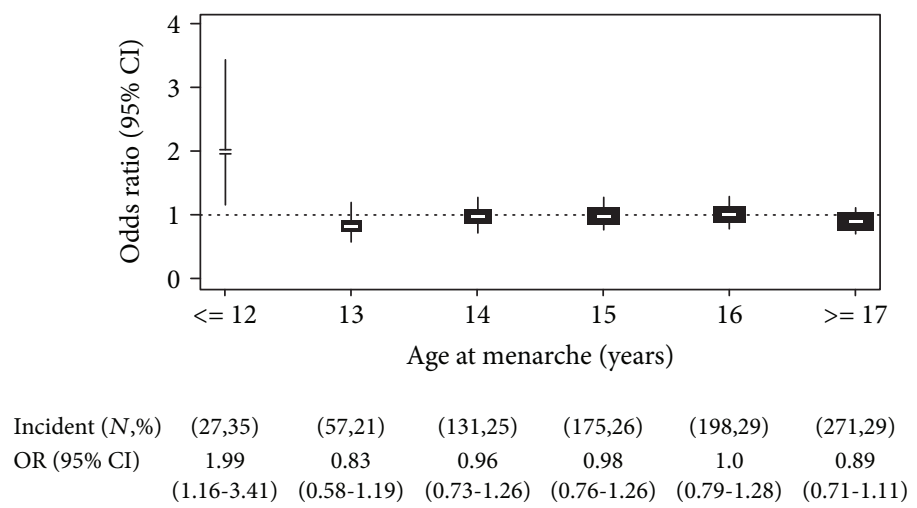

FIGURE 2: Risk of hypertriglyceridemia in each age at menarche. Odds ratio (OR) and 95\% confidence interval (CI) of hypertriglyceridemia by each age at menarche. $N$ is the number of incidence in each age of menarche; $\%$ is the percent of incidence in each age of menarche. The size of rectangle represents the number of samples. The vertical line indicates the overall OR on each age of menarche. ORs were adjusted for area, age, income, education, and C-reactive protein levels. The reference category was menarche at 16 years of age.

respective null hypotheses of the joint effects $\left(\beta_{\text {main }}=0\right.$ and $\left.\beta_{\text {int }}=0\right)$ and of the interaction effect $\left(\beta_{\text {int }}=0\right)$, respectively.

The critical $P$ values for accessing the significance of interaction were calculated by Bonferroni correction $\left(P<1.45 \times 10^{-7}\right)$ or the false discovery rate (FDR), with a $q$ value less than 0.05 . Quantile-quantile (QQ) plots of the $P$ values for the joint test and interaction analysis suggested a moderate inflation of the genome-wide analysis only for TG. The inflation factors were 1.32 and 1.24 for interaction and joint effects, respectively, for TG in KARE dataset (Supplemental Figure S2). There were no genomic inflations for other components $(\lambda<1.05)$. Therefore, for genomic controls of TG, the inverse normal transformed TG was used as a response, adjusting for age, area, income, education, and CRP levels for KARE. All analyses were performed using the $\mathrm{R}$ software version 2.11.1 (http://www.r-project.org/). Data management, descriptive statistics for the covariates and outcome variates, and the regression analyses were conducted using the Stats R package.

2.5. Pathway-Based Analysis. Pathway-based approaches using GWAS data are now used routinely to study complex diseases [29]. To analyze pathways interacting AAM with TG genetic polymorphism, we used the improved gene set enrichment analysis (GSEA) [29]. This approach has the advantage that genetic variant associations, mapping to any genes, provide insight into biological functions, signal pathways, and mechanisms of disease.

In our study, 344,396 SNPs were mapped to genes within $20 \mathrm{~kb}$ boundaries. Pathways consisting of $<20$ or $>200$ genes were excluded from further analysis, to reduce the multiple testing issue and avoid testing overly narrow or broad functional categories $[22,27]$. A false discovery rate (FDR) was used for multiple testing correction, with $q$ values $<0.05$ considered significant. Improved GSEA approaches use a comprehensive pathway and gene set database from SNP data. Pathway-related information is obtained from the KEGG (Kyoto Encyclopedia of Genes and Genomes pathway database), BioCarta, and GO (gene ontology) databases [27].

\section{Results}

3.1. Characteristics of Participants. A total of 3180 women (mean age: 56.07 years; SD: 8.88 years) were included in this study. Demographic characteristics of the women are presented in Table 1, which shows the baseline characteristics of the study population stratified by AAM. The mean age of menarche was 15.83 (SD of 1.89) years. Because of the small group numbers for each subject having earlier ages of menarche, we combined subjects aged 11 and 12 years of menarche. At baseline, women with earlier menarche were slightly younger, had higher BMI and higher alcohol consumption, and more frequently lived in urban areas (Table 1). The individuals with early menarche were more likely higher educated and had higher monthly incomes. However, we observed no association of OBS scores as an oxidative stress-related exposure and CRP levels as an inflammation marker with AAM. All women with early menarche had never regularly smoked.

3.2. Risks of Metabolic Syndrome and Its Association with Triglyceride by Age at Menarche. Figure 1 shows the ORs of MetS by each year of AAM (from 12 to 17 years). Compared with the reference group (age at 16 years), the risk of incident MetS was significantly increased only at $\leq 12$ years of AAM with 1.91 (95\% CI, 1.03-3.55, $P<0.05$ ), after adjustment for area, age, income, education, and CRP levels. We also investigated ORs between AAM and five individual components of MetS. Only hypertriglyceridemia was significantly increased at $\leq 12$ years of AAM with 1.99 (95\% CI, 1.16-3.41, $P<0.01$, Figure 2). However, other phenotypes did not significantly associate with AAM (Supplemental Figure S1).

We then examined the association between AAM and each component of MetS, separately. Linear regression analysis (Table 2) showed that women with earlier AAM had higher waist circumference and TG, compared with those with average AAM, after adjusting for the same covariates $(P<0.05)$. The corresponding regression coefficients of waist circumference and TG were $3.54(95 \%$ 


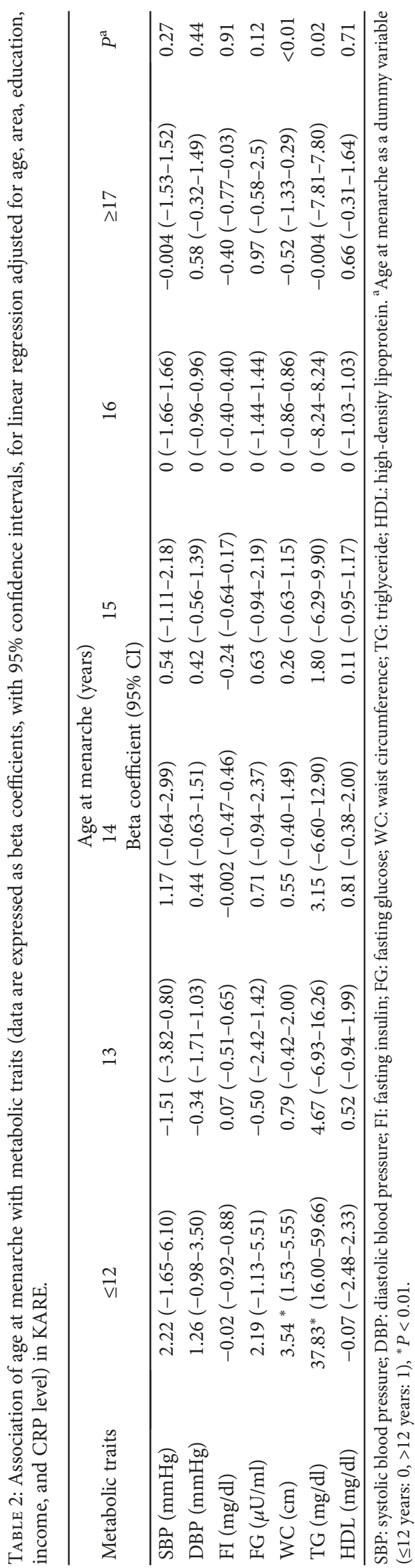


TABLE 3: Discovery GWAS top hits for joint and $1 \mathrm{df}$ interaction of gene age at menarche on triglyceride.

\begin{tabular}{|c|c|c|c|c|c|c|c|}
\hline \multirow{2}{*}{ SNP ID } & \multirow{2}{*}{ Nearest gene } & \multirow{2}{*}{$\mathrm{Chr}$} & \multirow{2}{*}{ Ref/var } & \multirow{2}{*}{ Position } & \multirow{2}{*}{ MAF } & \multicolumn{2}{|c|}{ Test of interaction } \\
\hline & & & & & & $P_{\text {joint }}$ & $P_{\text {int }}$ \\
\hline rs6589566 & ZPR1 & 11 & $\mathrm{~A} / \mathrm{C}$ & $116,781,707$ & 0.22 & $2.92 \times 10^{-12}$ & 0.15 \\
\hline rs 10503669 & $L P L$ & 8 & $\mathrm{~A} / \mathrm{C}$ & $19,990,179$ & 0.12 & $3.87 \times 10^{-9}$ & 0.03 \\
\hline rs603446 & ZPR1 & 11 & $\mathrm{C} / \mathrm{T}$ & $116,783,719$ & 0.23 & $4.82 \times 10^{-9}$ & 0.67 \\
\hline rs 17482753 & $L P L$ & 8 & $\mathrm{G} / \mathrm{T}$ & $19,975,135$ & 0.13 & $1.40 \times 10^{-8}$ & 0.02 \\
\hline rs17410962 & $L P L$ & 8 & $\mathrm{~A} / \mathrm{G}$ & $19,990,569$ & 0.13 & $1.48 \times 10^{-8}$ & 0.02 \\
\hline rs11216186 & SIK3 & 11 & $\mathrm{C} / \mathrm{T}$ & $116,913,976$ & 0.13 & $2.41 \times 10^{-8}$ & 0.03 \\
\hline rs17120157 & SIK3 & 11 & $\mathrm{C} / \mathrm{T}$ & $116,941,912$ & 0.13 & $6.43 \times 10^{-8}$ & 0.03 \\
\hline rs 10892068 & SIK3 & 11 & $\mathrm{C} / \mathrm{T}$ & $117,057,830$ & 0.13 & $7.34 \times 10^{-8}$ & 0.03 \\
\hline rs 17120293 & SIK3 & 11 & $\mathrm{C} / \mathrm{T}$ & $117,074,130$ & 0.13 & $7.44 \times 10^{-8}$ & 0.03 \\
\hline rs 12292858 & SIK3 & 11 & $\mathrm{~A} / \mathrm{C}$ & $116,943,263$ & 0.18 & $9.76 \times 10^{-8}$ & 0.09 \\
\hline rs2044426 & SIK3 & 11 & $\mathrm{C} / \mathrm{T}$ & $116,885,467$ & 0.10 & $1.52 \times 10^{-7}$ & 0.02 \\
\hline rs6073350 & JPH2 & 20 & $\mathrm{~A} / \mathrm{T}$ & $44,145,104$ & 0.04 & $1.61 \times 10^{-7}$ & 7.14 \\
\hline rs12279433 & SIK3 & 11 & $\mathrm{G} / \mathrm{T}$ & $116,877,505$ & 0.10 & $1.69 \times 10^{-7}$ & 0.02 \\
\hline rs1501675 & ARHGEF28 & 5 & $\mathrm{~A} / \mathrm{C}$ & $73,695,382$ & 0.02 & $1.77 \times 10^{-7}$ & $6.12 \times 10^{-8}$ \\
\hline rs1014492 & SCG2 & 2 & $\mathrm{~A} / \mathrm{G}$ & $223,633,665$ & 0.10 & $2.23 \times 10^{-7}$ & $4.49 \times 10^{-4}$ \\
\hline rs11216315 & PCSK7 & 11 & $\mathrm{C} / \mathrm{G}$ & $117,209,924$ & 0.13 & $2.94 \times 10^{-7}$ & 0.04 \\
\hline rs11216126 & BUD13 & 11 & $\mathrm{~A} / \mathrm{C}$ & $116,746,524$ & 0.20 & $3.60 \times 10^{-7}$ & 0.12 \\
\hline rs7513082 & LOC10537172 (UBQLN4) & 1 & $\mathrm{~A} / \mathrm{C}$ & $156,031,555$ & 0.02 & $4.01 \times 10^{-7}$ & $5.82 \times 10^{-8}$ \\
\hline rs13046 & TTT4 & 1 & $\mathrm{~A} / \mathrm{C}$ & $54,742,081$ & 0.01 & $6.18 \times 10^{-7}$ & $1.83 \times 10^{-5}$ \\
\hline rs 16875865 & LOC107986425 (LHFPL2) & 5 & $\mathrm{~A} / \mathrm{G}$ & $78,746,592$ & 0.01 & $6.40 \times 10^{-7}$ & $4.60 \times 10^{-7}$ \\
\hline
\end{tabular}

$\mathrm{CI}=1.53-5.55, P=0.01)$ and $37.83(95 \% \mathrm{CI}=16.00-59.66$, $P=0.02$ ), respectively. From these results (Figure 2 and Table 2), early menarche (12 years and before) is associated with increased risk of MetS, and this association appeared to be mediated mainly by increased TG of MetS.

3.3. Interaction between Genotypes and Age at Menarche on Triglyceride. As we considered that AAM was a modulator for the development of MetS, we conducted the joint analysis of the main SNP effect and its interaction effect with AAM interaction on MetS, and its individual traits, for all 352,228 SNPs. A list of SNPs associated with AAM-TG interactions, at $P<7 \times 10^{-7}$, by joint test, is provided in Table 3. The strongest statistical evidence for $\mathrm{SNP} \times \mathrm{AAM}$ joint interaction was an SNP of rs6589566, located on the chromosome 11, in zinc finger protein 1 (ZPR1) $\left(P=9.54 \times 10^{-7}\right)$. We found that main genetic effects of SNPs located chromosomes 11 and 8 were the primary contributors to these joint associations. Specifically, we identified SNP rs1501675, located within an intronic region of $A R H-$ GEF28, as the most significant SNP, with $P=6.12 \times 10^{-8}$ for AAM-to-TG. Other interesting SNPs were located near $( \pm 2 \mathrm{~kb})$ or within the loci LOC10537172, SCG2, TTT4, and LOC107986425 (all nominal $P$ values $<5 \times 10^{-4}$ ).

Based on these findings, we tested pathway level-based interaction between AAM and genetic variation, according to SNPs with significant $P$ values for enriched biological processes, in TG. When mapping SNPs were limited to $20 \mathrm{~kb}$ regions flanking a gene, three pathways, type 2 diabetes mellitus, stress-activated protein kinase (SAPK) signaling pathway, and Jun amino-terminal kinases (JNK) cascade, were significantly enriched, with association signals and FDR <0.05 (Table 4).

\section{Discussion}

In this study, we observed that early menarche (at or before 12 years of age) was associated with high prevalences of MetS; however, later AAM showed no significant association. In particular, this trend appeared to be mediated by increased TG, with earliest menarche ages associated with increased TG, in our study population. A number of studies have previously investigated the association between menarcheal age and MetS with elevated blood glucose or BMI or TG [30-32]. Recently, two studies have examined the association between AAM and risk of MetS in the Korean population. One study, of 1464 Korean women, showed that the relative risk of MetS was 3.84 (96\% CI: 1.52-9.70) for menarche at age $<12$ years in premenopausal women (from the KNHANES database) from 2007 to 2009, along with higher blood pressure [33]. Won et al. also recently reported that 12,336 participants with early menarche (age $<12$ years) were at increased risk of MetS $(\mathrm{OR}=1.35$, 95\% CI: $1.03-$ 2.12), with higher prevalence of hypertension and diabetes in KNHANES from 2010 to 2013 [19].

Puberty is the transition to adulthood that culminates in the production of mature gametes and the initiation of reproductive activity. The process begins within the central nervous system, where gonadotropin-releasing hormone $(\mathrm{GnRH})$ neurons are activated to release the neurohormone, stimulating pituitary gonadotropic hormone secretions that in turn direct gonadal steroid hormone production. 
TABLe 4: Pathway-based analysis of interaction between early menarche and genetic variation for triglyceride in Korean women.

\begin{tabular}{|c|c|c|c|c|c|}
\hline Pathways & Description & FDR & $\begin{array}{l}\text { Significant } \\
\text { genes }\end{array}$ & $\begin{array}{l}\text { Selected } \\
\text { genes }\end{array}$ & All genes \\
\hline Type 2 diabetes mellitus & KEGG type 2 diabetes mellitus & 0.016 & 17 & 33 & 44 \\
\hline $\begin{array}{l}\text { Stress-activated protein } \\
\text { kinase signaling pathway } \\
\text { (GO: 0031098) }\end{array}$ & Stress-activated protein kinase (SAPK) cascade & 0.019 & 14 & 38 & 49 \\
\hline $\begin{array}{l}\text { JNK cascade } \\
(\text { GO: } 0007254)\end{array}$ & $\begin{array}{l}\text { A cascade of protein kinase activities, culminating in } \\
\text { the phosphorylation and activation of a member of } \\
\text { the JUN kinase subfamily of stress-activated protein } \\
\text { kinases }\end{array}$ & 0.021 & 14 & 37 & 47 \\
\hline
\end{tabular}

Therefore, a younger AAM is associated with higher cumulative exposure to ovarian hormones [34]. Estrogen increases TG by promoting synthesis of TG in the liver and secreting TG into the circulation as very-low-density lipoprotein (VLDL) particles [35]. TG levels correlatively increase during pregnancy with estrogen-induced stimulated secretion of hepatic TG-rich lipoprotein [36]. However, the etiology of MetS, with regard to its association with TG, has yet to be unraveled completely with consideration of AAM.

Several studies have also demonstrated the importance of environmental triggers (including endogenous and exogenous exposure to hormone) in the development of chronic disease. Therefore, assay of gene-environmental interactions helps to understand the etiology of disease. Although several GWA studies have investigated AAM and breast cancer risk $[4,37]$, few GWA studies have focused on AAM and MetS with its components. Therefore, we conducted pathway analysis for investigating interaction of genetic variants and TG using GSEA. We found that T2 diabetes mellitus, stressactivated protein kinase, and JNK cascade pathways were associated with AAM on TG levels.

During puberty, there are proinflammatory and prooxidative changes and relative insulin resistance, which also play a role in the development of T2D $[38,39]$. Early puberty may be causally related to lower insulin sensitivity and inflammatory changes [40]. Reductions of insulin sensitivity and compensatory hyperinsulinemia are physiological during puberty, and this partly reflects the effects of increased growth hormone and IGF-1. Recent study reported that GnRH signaling may regulate T2D using pathway enrichment analysis [41]. However, it was reported that obesity drives metabolic risk in the prepubertal population rather than premature adrenarche [42]. More detailed mechanisms of the synergistic effects of early menarche and TG require further investigation.

SAPK/JNK are members of the MAPK family and are activated by a variety of environmental stresses, inflammatory cytokines, and growth factors. GnRH can activate the JNK/SAPK, p38, and ERK5 cascades in different cell models with varying kinetics [43]. In addition, JNKs may be a central mediator of impaired glucose metabolism and insulin resistance [44]. JNKs are also activated in obesity in numerous metabolically important cells and tissues such as adipose tissue, macrophages, the liver, skeletal muscle, and regions of the brain and pituitary. Recent studies have clearly established the important roles JNK signaling fulfils in macrophages, the liver, and cells of the anterior pituitary. Macrophage TG accumulation upregulates PON2 expression via the $\mathrm{MEK} / \mathrm{JNK} / \mathrm{c}$-Jun pathway, and these effects could be related, at least in part, to cellular TG-induced ROS formation [45]. Collectively, these studies place JNKs as important mediator of disruptions to metabolic homeostasis. In addition, 2-methoxyestradio activated SAPK/JNK in endothelial cells in a concentration-dependent manner [46]. Although several studies shed light on the associations of stress-related pathway and TG, assessing interaction of AAM is still challenging.

Our study provides some interesting results on the interplay between AAM and the genome for TG; however, some limitations need to be considered. One of them is the relatively small sample size of women with early menarche, which may provide a lack of statistical power, limiting the magnitude of interaction effects. In addition, another weakness could be the cross-sectional design of the study, with self-reported medical histories and recalled AAM. Even though AAM can be recalled by women with moderate accuracy, the use of self-reported menarcheal age may lead to some degree of recall bias [47], and recall can be influenced by the individual's current health condition [48]. Since TG might be associated with other chronic diseases such as cardiovascular disease or hyperlipidemia [49], we tried to investigate the association between TG and other chronic diseases such as hyperlipidemia and coronary artery disease (CAD) in the study participants. Based on self-reported data to chronic diseases, we could not investigate the association between TG and other chronic diseases such as hyperlipidemia and CAD due to this small sample size lack of information. Considering confounding factors on TG, we could not investigate the effect of lipid-lowering drug and estrogen use on TG due to the small size. Due to lack of relevant information, the confounding factors mentioned above are excluded from our analysis, which may limit the strength of our findings.

This is a first attempt of a genome-wide gene-AAM interaction study on MetS components in Korean women. We identified that type 2 diabetes mellitus, the SAPK signaling pathway, and JNK cascade were associated with TG including the genetic interaction with AAM. These findings may help us understand the role of AAM on the development of MetS and gene-environment interactions that confer MetS susceptibility. 


\section{Data Availability}

GWAS dataset and epidemiological data for KARE project are third party data and are available under the approval of the data access committee of the National Biobank of Korea, who can be contacted at biobank@korea.kr.

\section{Conflicts of Interest}

The authors declare that there is no conflict of interest regarding the publication of this paper.

\section{Acknowledgments}

This work was supported by the Bio \& Medical Technology Development Program of the National Research Foundation of Korea (NRF) grant (2013M3A9C4078158) and by grants of the Korea Health Technology R\&D Project through the Korea Health Industry Development Institute (KHIDI), funded by the Ministry of Health \& Welfare, Republic of Korea (grant numbers HI16C2037, HI15C2165 and HI16C2048).

\section{Supplementary Materials}

Supplemental Figure S1. Risk of components of MetS in each age of menarche. ORs are adjusted by area, age, income, education, and C-reactive protein levels. Reference category is menarche at 16 years of age. Size of rectangle represents number of samples. A: high blood pressure; B: high fasting glucose; C: abdominal obesity; D: low HDL cholesterol. Supplemental Figure S2. Quantile-quantile plot of observed $P$ values vs. expected $P$ values (on a $-\log 10$ scale) for joint and interaction analyses of SNP and age at menarche on TG. A: interaction test; B: joint test; C: interaction test by reverse normal distribution; D: joint test by reverse normal distribution. (Supplementary Materials)

\section{References}

[1] D. Charalampopoulos, A. McLoughlin, C. E. Elks, and K. K. Ong, "Age at menarche and risks of all-cause and cardiovascular death: a systematic review and meta-analysis," American Journal of Epidemiology, vol. 180, no. 1, pp. 29-40, 2014.

[2] A. Rudolph, J. Chang-Claude, and M. K. Schmidt, "Gene-environment interaction and risk of breast cancer," British Journal of Cancer, vol. 114, no. 2, pp. 125-133, 2016.

[3] S. Warren Andersen, A. Trentham-Dietz, R. E. Gangnon et al., "Breast cancer susceptibility loci in association with age at menarche, age at natural menopause and the reproductive lifespan," Cancer Epidemiology, vol. 38, no. 1, pp. 62-65, 2014.

[4] S. Warren Andersen, A. Trentham-Dietz, R. E. Gangnon et al., "The associations between a polygenic score, reproductive and menstrual risk factors and breast cancer risk," Breast Cancer Research and Treatment, vol. 140, no. 2, pp. 427-434, 2013.

[5] M. Ibitoye, C. Choi, H. Tai, G. Lee, and M. Sommer, "Early menarche: a systematic review of its effect on sexual and reproductive health in low- and middle-income countries," PLOS One, vol. 12, no. 6, article e0178884, 2017.

[6] N. Saquib, D. Kritz-Silverstein, and E. Barrett-Connor, "Age at menarche, abnormal glucose tolerance and type 2 diabetes mellitus: the Rancho Bernardo Study," Climacteric, vol. 8, no. 1, pp. 76-82, 2005.

[7] A. Gambineri, C. Pelusi, E. Manicardi et al., "Glucose intolerance in a large cohort of Mediterranean women with polycystic ovary syndrome: phenotype and associated factors," Diabetes, vol. 53, no. 9, pp. 2353-2358, 2004.

[8] M. Kivimèki, D. A. Lawlor, G. D. Smith et al., "Association of age at menarche with cardiovascular risk factors, vascular structure, and function in adulthood: the Cardiovascular Risk in Young Finns study," American Journal of Clinical Nutrition, vol. 87, no. 6, pp. 1876-1882, 2008.

[9] D. Stöckl, A. Döring, A. Peters et al., "Age at menarche is associated with prediabetes and diabetes in women (aged 32-81 years) from the general population: the KORA F4 study," Diabetologia, vol. 55, no. 3, pp. 681-688, 2012.

[10] R. Lakshman, N. Forouhi, R. Luben et al., "Association between age at menarche and risk of diabetes in adults: results from the EPIC-Norfolk cohort study," Diabetologia, vol. 51, no. 5, pp. 781-786, 2008.

[11] L. Ibáñez, A. Lopez-Bermejo, M. Diaz, M. V. Marcos, and F. de Zegher, "Early metformin therapy to delay menarche and augment height in girls with precocious pubarche," Fertility and Sterility, vol. 95, no. 2, pp. 727-730, 2011.

[12] J. Kaur, "A comprehensive review on metabolic syndrome," Cardiology Research and Practice, vol. 2014, Article ID 943162, 21 pages, 2014.

[13] S. O'Neill and L. O'Driscoll, "Metabolic syndrome: a closer look at the growing epidemic and its associated pathologies," Obesity Reviews, vol. 16, no. 1, pp. 1-12, 2015.

[14] C. L. Avery, Q. He, K. E. North et al., "A phenomics-based strategy identifies loci on APOC1, BRAP, and PLCG1 associated with metabolic syndrome phenotype domains," PLoS Genetics, vol. 7, no. 10, article e1002322, 2011.

[15] T. M. Teslovich, K. Musunuru, A. V. Smith et al., "Biological, clinical and population relevance of 95 loci for blood lipids," Nature, vol. 466, no. 7307, pp. 707-713, 2010.

[16] R. J. Khan, S. Y. Gebreab, M. Sims, P. Riestra, R. Xu, and S. K. Davis, "Prevalence, associated factors and heritabilities of metabolic syndrome and its individual components in African Americans: the Jackson Heart Study," BMJ Open, vol. 5, no. 10, article e008675, 2015.

[17] C. M. Povel, J. M. A. Boer, and E. J. M. Feskens, "Shared genetic variance between the features of the metabolic syndrome: heritability studies," Molecular Genetics and Metabolism, vol. 104, no. 4, pp. 666-669, 2011.

[18] Y. Kim, B. G. Han, and the KoGES group, "Cohort profile: the Korean Genome and Epidemiology Study (KoGES) consortium," International Journal of Epidemiology, vol. 46, no. 2, article e20, 2017.

[19] J. C. Won, J. W. Hong, J. H. Noh, and D. J. Kim, “Association between age at menarche and risk factors for cardiovascular diseases in Korean women: the 2010 to 2013 Korea National Health and Nutrition Examination Survey," Medicine, vol. 95, no. 18, article e3580, 2016.

[20] J. Kim, J. Kim, H. Min et al., "Joint identification of genetic variants for physical activity in Korean population," International Journal of Molecular Sciences, vol. 15, no. 7, pp. 12407-12421, 2014.

[21] T. H. Baek, H. Y. Lee, N. K. Lim, and H. Y. Park, "Gender differences in the association between socioeconomic status and hypertension incidence: the Korean Genome and 
Epidemiology Study (KoGES)," BMC Public Health, vol. 15, no. 1, 2015.

[22] H. S. Lee and T. Park, "Pathway-driven approaches of interaction between oxidative balance and genetic polymorphism on metabolic syndrome," Oxidative Medicine and Cellular Longevity, vol. 2017, Article ID 6873197, 9 pages, 2017.

[23] S. Lakkur, S. Judd, R. M. Bostick et al., "Oxidative stress, inflammation, and markers of cardiovascular health," Atherosclerosis, vol. 243, no. 1, pp. 38-43, 2015.

[24] P. Zimmet, D. Magliano, Y. Matsuzawa, G. Alberti, and J. Shaw, "The metabolic syndrome: a global public health problem and a new definition," Journal of Atherosclerosis and Thrombosis, vol. 12, no. 6, pp. 295-300, 2005.

[25] J. C. Bae, N. H. Cho, S. Suh et al., "Cardiovascular disease incidence, mortality and case fatality related to diabetes and metabolic syndrome: a community-based prospective study (Ansung-Ansan cohort 2001-12)," Journal of Diabetes, vol. 7, no. 6, pp. 791-799, 2015.

[26] Y. S. Cho, M. J. Go, Y. J. Kim et al., “A large-scale genome-wide association study of Asian populations uncovers genetic factors influencing eight quantitative traits," Nature Genetics, vol. 41, no. 5, pp. 527-534, 2009.

[27] H. S. Lee and T. Park, "Nuclear receptor and VEGF pathways for gene-blood lead interactions, on bone mineral density, in Korean smokers," PLoS One, vol. 13, no. 3, article e0193323, 2018.

[28] X. Y. Wu, H. Cai, A. Kallianpur et al., "Age at menarche and natural menopause and number of reproductive years in association with mortality: results from a median follow-up of 11.2 years among 31, 955 naturally menopausal Chinese women," PLoS One, vol. 9, no. 8, article e103673, 2014.

[29] K. Zhang, S. Cui, S. Chang, L. Zhang, and J. Wang, " $i$ GSEA4GWAS: a web server for identification of pathways/gene sets associated with traits by applying an improved gene set enrichment analysis to genome-wide association study," Nucleic Acids Research, vol. 38, Supplement 2, pp. W90W95, 2010.

[30] M. Heys, C. M. Schooling, C. Jiang et al., "Age of menarche and the metabolic syndrome in China," Epidemiology, vol. 18, no. 6, pp. 740-746, 2007.

[31] D. Stöckl, C. Meisinger, A. Peters et al., "Age at menarche and its association with the metabolic syndrome and its components: results from the KORA F4 study," PLoS One, vol. 6, no. 10, article e26076, 2011.

[32] S. Akter, S. Jesmin, M. Islam et al., "Association of age at menarche with metabolic syndrome and its components in rural Bangladeshi women," Nutrition \& Metabolism, vol. 9, no. 1, p. 99, 2012.

[33] Y. J. Cho and G. H. Lee, "Association of age at menarche with metabolic syndrome and components of metabolic syndrome in premenopausal women, Korea National Health and Nutrition Examination Survey VI," International Journal of Diabetes in Developing Countries, vol. 36, no. 3, pp. 345351, 2016.

[34] O. Y. Addo, B. S. Miller, P. A. Lee, M. L. Hediger, and J. H. Himes, "Age at hormonal onset of puberty based on luteinizing hormone, inhibin $\mathrm{B}$, and body composition in preadolescent US girls," Pediatric Research, vol. 76, no. 6, pp. 564-570, 2014.

[35] J. Lee and I. J. Goldberg, "Hypertriglyceridemia-induced pancreatitis created by oral estrogen and in vitro fertilization ovulation induction," Journal of Clinical Lipidology, vol. 2, no. 1, pp. 63-66, 2008.

[36] L. Berglund, J. D. Brunzell, A. C. Goldberg et al., "Evaluation and treatment of hypertriglyceridemia: an Endocrine Society clinical practice guideline," The Journal of Clinical Endocrinology and Metabolism, vol. 97, no. 9, pp. 2969-2989, 2012.

[37] M. V. Coignet, G. R. Zirpoli, M. R. Roberts et al., "Genetic variations, reproductive aging, and breast cancer risk in African American and European American women: The Women's Circle of Health Study," PLoS One, vol. 12, no. 10, article e0187205, 2017.

[38] R. P. Mathew, D. W. Byrne, M. R. F. Linton, D. E. Vaughan, S. Fazio, and W. E. Russell, "Evidence of metabolic syndrome in lean children with premature pubarche at diagnosis," Metabolism, vol. 57, no. 6, pp. 733-740, 2008.

[39] L. Ibanez, K. Ong, F. de Zegher, M. V. Marcos, L. del Rio, and D. B. Dunger, "Fat distribution in non-obese girls with and without precocious pubarche: central adiposity related to insulinaemia and androgenaemia from prepuberty to postmenarche," Clinical Endocrinology, vol. 58, no. 3, pp. 372-379, 2003.

[40] D. A. Wilson, J. G. B. Derraik, D. L. Rowe, P. L. Hofman, and W. S. Cutfield, "Earlier menarche is associated with lower insulin sensitivity and increased adiposity in young adult women," PLoS One, vol. 10, no. 6, article e0128427, 2015.

[41] W. Xiang, B. Zhang, F. Lv et al., "The potential regulatory mechanisms of the gonadotropin-releasing hormone in gonadotropin transcriptions identified with bioinformatics analyses," Reproductive Biology and Endocrinology, vol. 15, no. 1, p. 46, 2017.

[42] K. M. Williams, S. E. Oberfield, C. Zhang, D. J. McMahon, and A. B. Sopher, "The relationship of metabolic syndrome and body composition in children with premature adrenarche: is it age related?," Hormone Research in Poediatrics, vol. 84, no. 6, pp. 401-407, 2015.

[43] R. M. Perrett and C. A. McArdle, "Molecular mechanisms of gonadotropin-releasing hormone signaling: integrating cyclic nucleotides into the network," Frontiers in Endocrinology, vol. 4, p. 180, 2013.

[44] M. Pal, M. A. Febbraio, and G. I. Lancaster, "The roles of c-Jun NH2-terminal kinases (JNKs) in obesity and insulin resistance," The Journal of Physiology, vol. 594, no. 2, pp. 267-279, 2016.

[45] M. Rosenblat, N. Volkova, N. Paland, and M. Aviram, "Triglyceride accumulation in macrophages upregulates paraoxonase 2 (PON2) expression via ROS-mediated JNK/c-Jun signaling pathway activation," BioFactors, vol. 38, no. 6, pp. $458-469,2012$.

[46] T. L. Yue, X. Wang, C. S. Louden et al., "2-Methoxyestradiol, an endogenous estrogen metabolite, induces apoptosis in endothelial cells and inhibits angiogenesis: possible role for stress-activated protein kinase signaling pathway and Fas expression," Molecular Pharmacology, vol. 51, no. 6, pp. 951962, 1997.

[47] A. Bergsten-Brucefors, "A note on the accuracy of recalled age at menarche," Annals of Human Biology, vol. 3, no. 1, pp. 7173, 2009.

[48] R. Cooper, M. Blell, R. Hardy et al., "Validity of age at menarche self-reported in adulthood," Journal of Epidemiology and Community Health, vol. 60, no. 11, pp. 993-997, 2006.

[49] B. G. Nordestgaard and A. Varbo, "Triglycerides and cardiovascular disease," Lancet, vol. 384, no. 9943, pp. 626-635, 2014. 


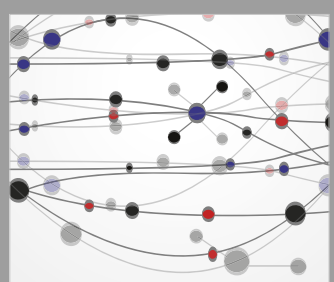

The Scientific World Journal
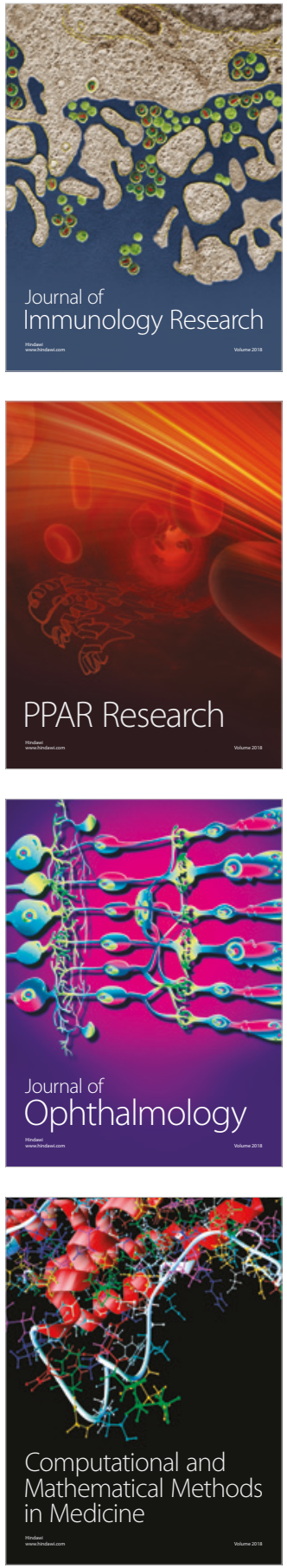

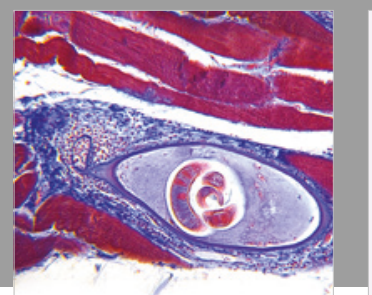

Gastroenterology Research and Practice

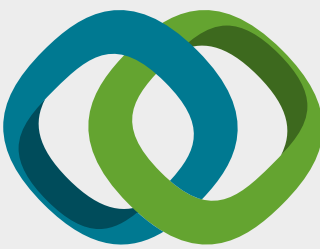

\section{Hindawi}

Submit your manuscripts at

www.hindawi.com
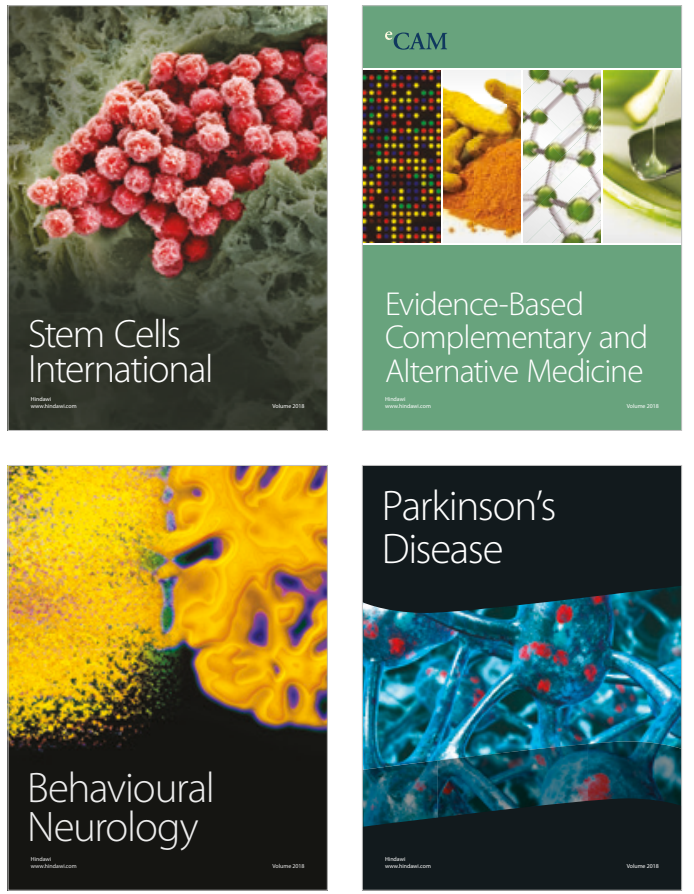

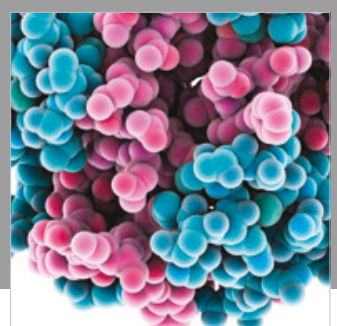

ournal of

Diabetes Research

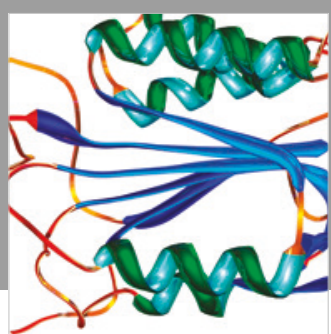

Disease Markers
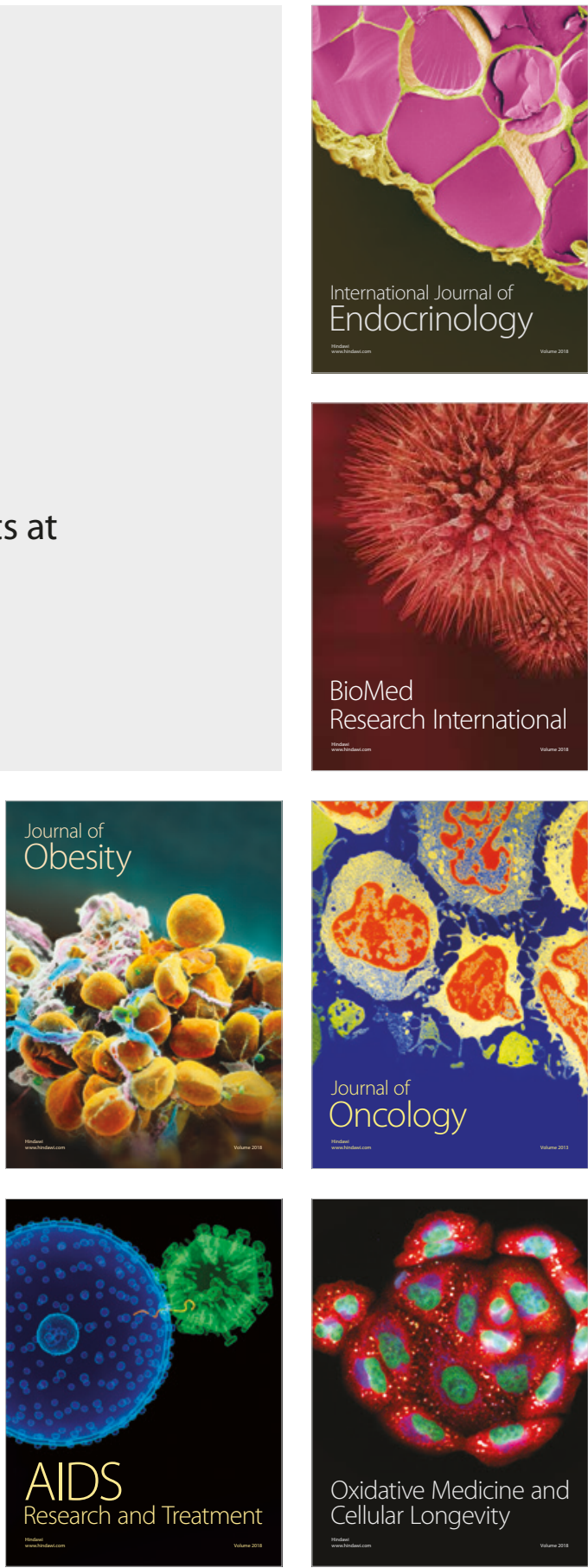